Michel Grossetti, Centre Interdisciplinaire d'Études Urbaines (CIEU), Université de Toulouse le Mirail, 5, allées A. Machado, 31058 Toulouse Cedex, tel : 05615036 69, fax : 056150 49 61, E-Mail : rgros@cict.fr

\title{
Communication électronique et réseaux sociaux ${ }^{1}$
}

\author{
Flux, n²9, Juillet 1998, pp.5-13
}

\section{Résumé :}

L'analyse des réseaux sociaux est un champ de recherche important qui s'intéresse aux formes de communication dans la mesure où celles-ci interviennent dans la création ou le maintien des relations sociales. L'émergence de la communication électronique suscite dans ce cadre des interrogations spécifiques concernant l'évolution de la structure des réseaux suscitée par l'accès de plus en plus répandu à ce type de moyen. l'étude des correspondants réguliers d'un échantillon de chercheurs toulousains nous permet de saisir certaines de ces évolutions. Comme on pouvait s'y attendre, le rôle de la proximité dans la création et le maintien des relations sociales s'affaiblit. Les réseaux sociaux fondés sur un usage important de la communication électronique présentent des spécificités structurelles intéressantes qui les différencient des réseaux "ordinaires" par des traits similaires à ceux qui spécifient les réseaux sociaux des grandes villes par rapport aux réseaux en milieu moins urbanisé. Ce résultat suggère l'hypothèse de l'"hyper-ville" qui fait de la généralisation de la communication électronique une sorte d'équivalent de l'urbanisation, du moins en ce qui concerne les relations sociales.

\section{Présentation de l'auteur :}

Michel Grossetti est chercheur au CNRS et centre ses recherches sur la proximité spatiale dans les activités scientifiques et les rapports que celles-ci entretiennent avec leur environnement (il a publié l'ouvrage Science, Industrie et Territoire, aux Presses Universitaires du Mirail en 1995). Il s'intéresse plus généralement à la proximité dans les réseaux sociaux.

\footnotetext{
${ }^{1}$ Ce texte présente les résultats d'une recherche réalisée dans le cadre du DEA de Sociologie de l'Université de Toulouse-le-Mirail avec Anne Sauvageot et ses étudiants : Patrick Burtin, David Merle, Véréna Paravel, Jean-Luc Védrines, Patricia Malgoyre, Pierre Houdeau et Lionel Mazoyer. Cette recherche a par ailleurs bénéficié de la collaboration de Joseph Saint Pierre, ingénieur au Centre interuniversitaire de calcul de Toulouse (CICT).
} 
Le développement de la communication électronique, en particulier le courrier interindividuel et les groupes de discussion, interroge fortement depuis quelques années l'analyse des réseaux sociaux en faisant évoluer les cadres d'interaction, donc le fondement même de la formation et de l'entretien des relations sociales.

Rappelons que l'analyse des réseaux sociaux est une approche des phénomènes sociaux qui s'est progressivement formalisée à partir des années cinquante en s'appuyant sur certains travaux précurseurs de Simmel ou de l'anthropologie britannique. Cette approche prend pour objet premier les relations sociales concrètes et reconstruit les structures collectives à partir de l'observation et l'analyse de ces relations et des réseaux qu'elles constituent. Elle s'oppose ainsi méthodologiquement aussi bien aux théories holistes privilégiant les catégories (âge, sexe, classe sociale, etc.) qu'aux modèles individualistes étudiant l'agrégation de comportements individuels supposés rationnels. L'analyse des réseaux sociaux s'est dotée de méthodes et de notions spécifiques d'analyse formelle des réseaux (densité, polyvalence et force des liens, etc.) et a progressivement accumulé un certain nombre de résultats sur la façon dont la structure des réseaux dans lesquels les acteurs sont insérés est à la fois une contrainte et une ressource pour l'action ${ }^{2}$.

Jusqu'à présent, l'analyse des réseaux sociaux s'est peu interrogée sur les moyens de communication et l'influence que peut avoir leur évolution sur les modes de construction et de fonctionnement des réseaux sociaux. Ce champ de recherche s'étant construit dans un monde connaissant déjà depuis longtemps le téléphone, on peut penser que c'est la relative stabilité des moyens techniques d'interaction qui explique ce désintérêt. L'apparition de la télécopie par exemple n'a pas suscité à notre connaissance d'étude particulière de la part des spécialistes de l'analyse des réseaux sociaux, probablement parce qu'elle ne modifie pas fondamentalement le fonctionnement des relations interindividuelles. Par contre, le développement de la communication électronique, et singulièrement d'Internet, à commencé à attirer l'attention de certains chercheurs du domaine, peut-être parce que ce dispositif de communication touche fortement la communauté scientifique ${ }^{3}$. Le récent colloque annuel de cette communauté de recherche (San Diego, Mars 1997) comportait trois sessions consacrées aux rapports entre réseaux électroniques et réseaux sociaux (intitulées "Computer Networks as Social Networks"), toutes trois animées par Barry Wellman, directeur du Centre for Urban and Community Studies de l'Université de Toronto.

En s'appuyant à la fois sur les travaux publiés depuis une quinzaine d'années en psychologie sociale ou en sciences de la communication, ainsi que sur leurs propres travaux ${ }^{4}$, Wellman et son équipe ont clairement formulé dans des publications récentes ${ }^{5}$

\footnotetext{
${ }^{2}$ Un certain nombre d'ouvrages présentent l'analyse de réseaux de façon évidemment plus complète que ce rappel très succinct : A. Degenne et M. Forsé, 1994, Les Réseaux Sociaux, Paris, Armand Colin ; J. Scott, 1992, Social Network Analysis, Newbury Park CA, Sage ; S. Wasserman and K. Faust, 1994, Social Network Analysis, Cambridge, Cambridge University Press ; Barry Wellman and S.D. Berkowitz, 1997, Social Structures : A Network Approach, Greenwich, CT JAI Press.

${ }^{3}$ Le réseau international des analystes de réseaux sociaux (International Network for Social Network Analysis, INSNA) s'est évidemment très vite doté d'un site web (http://www.heinz.cmu.edu/project/INSNA/) et d'une liste de distribution (SOCNET).

4 Une essentiellement, portant sur les communications des chercheurs, par le réseau et en dehors (Caroline Haythornwaite et Barry Wellman, 1996, "Which kinds of network members communicate by Email or face-to-face for what kind of work ?", University of Toronto, Centre for urban and community studies, 10p.)
} 
un certain nombre des questions que pose le développement de la communication électronique à l'analyse des réseaux sociaux. Après avoir argumenté l'intérêt d'étudier empiriquement les réseaux électroniques du point de vue de l'analyse des réseaux sociaux $^{6}$, Wellman et son équipe montrent que le courrier électronique tend à gommer les différences de statut social et encourage les comportements non conformistes, permettant dans les échanges de groupe une implication plus large et plus égalitaire des participants. Il peut produire des liens forts ${ }^{7}$ (des personnes peuvent se "rencontrer" sur le réseau et construire une relation forte), mais supporte en général plutôt des liens relativement faibles, informels, à interaction fréquente, utiles et spécialisés ${ }^{8}$, ce qu'ils désignent comme des «relations intimes secondaires» ("intimate secondary relationships »). Sur le plan de la structure des réseaux sociaux le développement de la communication électronique aurait pour effet d'accroître la taille des réseaux individuels (le nombre de liens), leur dispersion dans l'espace, et le nombre de groupes différents avec lesquel une même personne peut échanger. Enfin, Wellman et son équipe établissent une liste des questions importantes à résoudre concernant les modalités d'usage du réseau, la façon dont la communication électronique se combine avec d'autres types de communication (téléphone, etc.), la densité ${ }^{9}$ des réseaux supportés par ordinateur, les modes de création, maintien ou disparition des relations électroniquement entretenues, le nombre et les caractéristiques sociales des usagers d'Internet, la "taille-réseau" d'Internet ${ }^{10}$, les possibilités de mobilisation politique par le réseau, etc. Il s'agit en fait de reprendre l'ensemble des questions (et des résultats) de l'analyse de réseau en intégrant le paramètre supplémentaire que représente l'accès à la communication électronique.

En touchant les cadres mêmes de l'interaction, la communication électronique contraint donc l'analyse des réseaux à réouvrir la boîte noire de l'interaction et de ses ingrédients techniques. Wellman et son équipe ont commencé à le faire en s'appuyant sur des

\footnotetext{
${ }^{5}$ Laura Garton et Barry Wellman, 1995, "Social impacts of electronic mail in organizations : a review of the research literature", in Brant R. Burleson (ed.) Communication yearbook, $\mathrm{n}^{\circ} 18$, Thousand Oaks, CA : Sage ; Barry Wellman, Janet Salaff, Dimitrina Dimitrova, Laura Garton, Milena Gulia, Caroline Haythornwaite, 1996, "Computer networks as social networks : collaborative work, telework, and virtual community", Annual review of Sociology, n²2 ; Barry Wellman et Milena Gulia, 1996 "Net surfers don't ride alone : virtual communities as communities", in P. Kollock and M. Smith (eds.), Communities in cyberspace, Berkeley, University of California Press ; Barry Wellman, 1996, "An electronic group is virtually a social network" in sara Kiesler (ed.), The culture of the Internet, Hillsdale, NJ : Lawrence Erlbaum.

${ }^{6}$ « Dans la mesure où les réseaux électroniques relient des gens autant que des machines, ils deviennent des réseaux sociaux, que nous appelons des réseaux sociaux assistés par ordinateur [computer-supported social networks, (CSSNs)]», Barry Wellman, et alii, 1996, "Computer networks as social networks : collaborative work, telework, and virtual community", op. cit., p.214

${ }^{7} \mathrm{La}$ force du lien est une dimension classique des relations sociales, qui résume le degré d'intimité, de fréquence des échanges, de confiance entre les participants. Un résultat classique de l'analyse de réseaux est que les liens faibles sont plus efficaces que les liens forts pour accéder à certaines ressources (trouver un emploi par exemple) (Mark Granovetter, 1973. "The strength of weak ties." American Journal of Sociology, Vol. 78).

${ }^{8}$ La spécialisation (ou son contraire la polyvalence) est une autre dimension importante des relations sociales : elle désigne la plus ou moins grande variété du contenu des échanges (professionnel, politique, concernant tel ou tel type d'activité, etc.).

${ }^{9}$ La densité désigne le nombre de liens activés par rapport au nombre de liens possibles pour un réseau donné.

10 Cette question fait référence au problème du "petit monde" posé par Milgram en 1967 ("The smallworld Problem", Psychology today, $\mathrm{n}^{\circ} 1$ ) dont l'expérience montrait qu'aux Etats-Unis, une moyenne de cinq relations interpersonnelles enchaînées permettait d'atteindre un membre quelconque de la population américaine.
} 
travaux de psychologie sociale et de sociologie de la communication américaines, mais montrent aussi la quasi-absence d'études empiriques spécifiquement orientées vers l'analyse des réseaux.

En France la communication électronique a fait l'objet de diverses études empiriques menées quasi-simultanément. Une équipe du CNET a réalisé une étude sur les usages $\mathrm{d}^{\prime I n t e r n e t}$ dans un centre de recherche industrielle ${ }^{11}$. Cette étude met en évidence deux types d'utilisateurs, les "internautes militants" et les "utilitaristes" et montre que l'usage d'Internet menace d'autres médias comme la télécopie ou même une partie de la presse spécialisée. Par ailleurs, selon cette étude, dans le contexte spécifique d'une entreprise privée, Internet tend à entrer en contradiction avec l'organisation hiérarchique et les contraintes de sécurité, notamment en brouillant les frontières entre travail et horstravail. Une autre étude ${ }^{12}$, sur l'usage d'Internet par des chercheurs strabourgeois, aborde entre autres la question du lien social sur le réseau, montrant en quoi les échanges électroniques redoublent et renforcent les liens existants. Cette étude examine aussi comment Internet peut être un support pour une écriture collective à travers les possibilités offertes d'enchaînements de messages, réponses, réponses à la réponse, etc.

Notre équipe a réalisé en 1995 une étude empirique de l'usage d'Internet par les chercheurs toulousains ${ }^{13}$. Utilisant plusieurs angles d'approche (analyse des flux de messages envoyés par les usagers du principal serveur toulousain; suivi des groupes de discussion locaux ; questionnaire envoyé par courrier électronique à tous les usagers scientifiques de la partie régionale de RENATER), nous avons pu établir un premier tableau global de l'usage du réseau par les chercheurs. Nous avons découvert que la majorité des messages ont pour destinataire une personne travaillant dans la même agglomération, voire la même université, mais que $90 \%$ des usagers ont au moins un correspondant à l'étranger et qu'une part significative des relations ont été initiées par échange électronique. La variété des services disponibles (courrier, groupes de discussion, Web, transfert de fichiers, communication interactive, etc.) définit différents types d'usages et d'usagers qui n'ont pas le même rapport à ce type de communication. Nous distinguions ainsi les "professionnels" (ayant totalement intégré les divers usages $\mathrm{du}$ réseau à leur pratique professionnelle mais n'en faisant pas ou peu d'usage non professionnel), les "communautaires" (utilisant les diverses possibilités du réseau tant dans leur exercice professionnel que pour d'autres aspects de leurs activités), les "minimalistes" (n'utilisant que le courrier électronique), les "utilitaristes" (n'utilisant que certains aspects très précis du réseau — transfert de fichier en particulier — pour répondre à un besoin spécifique) et les "contraints" (amenés à utiliser divers aspects du réseau sous la contrainte de leur environnement professionnel). Cette enquête montrait aussi que la dimension la plus importante du réseau, outre l'accès à l'information, est bien la construction et le maintien des relations sociales, ce qui rejoint le point de vue défendu par l'équipe de Wellman.

Plus récemment, nous avons amorcé une seconde recherche, plus centrée sur les réseaux sociaux, pour laquelle nous avons conçu une méthode adaptée de recueil d'information.

${ }^{11}$ F. Carmagnat, "Une société électronique technicienne face à l'élargissement du réseau", Réseaux, n77, CNET, 1996

12 P. Hert, "Les arts de lire le réseau. Un cas d'innovation technologique et ses usages au quotidien dans les sciences", Réseaux, n77, CNET, 1996

13 M. Grossetti, A. Sauvageot, Stéphane Branquart, Didier Marinesque, Céline Compère, Lucien Paganelle, Jean-Philippe Estebenet, Joseph Saint-Pierre, 1996, "L'usage d'Internet par les chercheurs toulousains", Flux, n²4, Avril-Juin 
Il s'agit d'entretiens longs réalisés sur les lieux de travail. Chaque enquêté ${ }^{14}$ devait ouvrir sa boîte aux lettres électronique et répondre à certain nombre de questions sur un échantillon d'une vingtaine de correspondants réguliers (avec lesquels les interactions dépassent un simple échange ponctuel). 15 entretiens ont été réalisés ${ }^{15}$, générant une population de 248 correspondants.

Les résultats de cette enquête nous permettent de revenir sur certains résultats de notre précédente étude, en particulier en ce qui concerne la localisation des correspondants, mais aussi d'aborder certaines des questions relatives aux réseaux sociaux. Nous disposons en effet dans ces données d'informations sur la densité des liens (nous demandions aux enquêtés de décrire les liens entre leurs correspondants), leur force, la fréquence des échanges, etc. Autrement dit, après une enquête exploratoire un peu générale, cette seconde investigation nous permet de commencer à traiter (encore insuffisamment à cause de l'étroitesse de notre échantillon d'enquêtés) la question des interactions entre les réseaux sociaux et les usages du courrier électronique. Pour cela il est intéressant de comparer les résultats de cette étude à deux enquêtes de référence sur les réseaux sociaux en milieu urbain : celle de Fisher (1982) sur la Californie et celle de Wellman (1979) sur Toronto. Ces deux enquêtes ont été réalisées avec une méthodologie comparable consistant à interroger un important échantillon de personnes (1050 pour Fisher, 845 pour Wellman) sur leurs relations identifiées dans un cas (Fisher) à partir d'un certain nombre de questions génératrices de noms ("Avec qui parlez vous de décisions concernant votre travail ?" ; "A qui confieriez-vous votre maison (arroser les plantes, relever le courrier) si vous vous absentiez ?" ; etc.), et dans l'autre cas à partir d'une question sur les six personnes dont les enquêtés se sentent les plus proches. Dans le premier cas, les réseaux ainsi constitués comptent en moyenne un peu plus de 18 personnes, dans le second ils en comportent au maximum six.

\section{Le nombre et la localisation des correspondants}

Lors de notre précédente étude, nous avions demandé aux enquêtés d'évaluer le nombre de leurs correspondants. $85 \%$ en déclaraient moins de 80 et $15 \%$ plus de 90 . Nous retrouvons à peu près ces proportions chez nos 15 enquêtés. Mais il faut distinguer les correspondants singuliers de ceux qui appartiennent aux listes collectives de distribution auxquelles sont abonnés les enquêtés. Lorsque le nombre de correspondants devient élevé, c'est en général parce que les listes de distribution y occupent une place importante et que les correspondants singuliers ne sont plus que ponctuels. Faute de temps, nous n'avons pu examiner en détail ce point, nous contentant de recueillir des informations sur un échantillon d'une vingtaine de correspondants. C'est donc sur ce sous ensemble de correspondants que portent les résultats qui suivent.

Lors de la précédente enquête nous avions été surpris par l'importance de la part locale des flux de messages, qui atteignait les trois quart d'un échantillon de 4880 messages transitant par le principal serveur universitaire toulousain (le reste de la France représentant un dixième et les pays étrangers un peu moins d'un cinquième). Même en tenant compte des messages internes au serveur générés automatiquement par la

\footnotetext{
14 les personnes interrogées ont été choisies parmis la population ayant répondu à notre première enquête de façon à reprséneter les différentes classes d'utilisateurs d'Internet que nous avions constituées.

156 chercheurs (CNRS ou INRIA), 6 enseignants-chercheurs, 3 étudiants de thèse. 4 "communautaires", 4 "professionnels", 4 "utilitaristes", 2 "minimalistes" et un "contraint". 11 hommes et 4 femmes.
} 
machine, nous avions évalué à plus de 50\% la part locale des messages envoyés par les utilisateurs de ce serveur. Il est intéressant de signaler qu'une mesure équivalente effectuée dans les mêmes conditions sur une même durée de temps (deux journées ouvrables) exactement un an plus tard montre un fort accroissement des flux (10410 messages) mais donne une répartition géographique assez similaire : $63 \%$ de messages à destination d'un correspondant toulousain ou de la région (dont $53 \%$ sur le même serveur), 10,5\% en France en dehors de la région Midi-Pyrénées et 26,5\% de messages vers l'étranger ${ }^{16}$.

Si nous examinons à présent la façon dont se répartissent géographiquement les 248 correspondants de nos 15 enquêtés, nous obtenons une vision nettement différente puisque $27 \%$ seulement sont à Toulouse, $30 \%$ ailleurs en France et $43 \%$ à l'étranger. Les correspondants locaux sont donc minoritaires. La différence entre les deux répartitions s'explique un peu par les messages "artificiels" générés par la machine mais surtout par la fréquence des échanges, qui varie en fonction inverse de la distance entre correspondants $(55 \%$ d'échanges au moins hebdomadaires pour les correspondants locaux contre moins de $25 \%$ pour les autres) ainsi que par la définition de notre population, qui ignore les correspondants occasionnels.

La notion de correspondants réguliers ne recouvre pas le type de lien étudié par Fisher (1982) ou de liens "intimes" étudiés par Wellman (1978). Par ailleurs, nous ne disposons que des liens entretenus par courrier électronique et non de la totalité du réseau. Rappelons toutefois que dans ces deux enquêtes de référence, la part "locale" (même agglomération ou moins d'une heure de transport) atteignait $70 \%$ pour Fisher et $75 \%$ pour Wellman. Dans notre enquête, cette part locale ne varie guère lorsqu'on ne prend en compte que des liens forts (elle avoisine les 33\%). Ce résultat semble indiquer que l'accès au courrier électronique peut s'accompagner d'une relative déterritorialisation des relations. Il faut toutefois être prudent : notre échantillon de base est étroit et ne concerne que des chercheurs, enseignants chercheurs ou étudiants de troisième cycle, soit une population spécifique en termes de réseau.

Le courrier électronique occupe une place centrale dans les mode de communication des personnes interrogées. Il est le mode le plus fréquent avec $30 \%$ des correspondants locaux, mais $89 \%$ des correspondants nationaux et $94 \%$ des correspondants à l'étranger. L'importance de sa place dans les dispositifs de communication ressort aussi de l'examen des types alternatifs de communication.

\begin{tabular}{|c|c|c|c|c|}
\hline $\begin{array}{l}\text { Localisation des } \\
\text { correspondants } \\
\text { Mode d'échange le } \\
\text { plus fréquent en dehors } \\
\text { du courrier électronique }\end{array}$ & Toulouse & France & Etranger & Total \\
\hline Face à face & $87 \%$ & $56 \%$ & $61 \%$ & $68 \%$ \\
\hline
\end{tabular}

${ }^{16}$ Les deux études de flux ont été réalisées par Joseph Saint Pierre, ingénieur au Centre interuniversitaire de calcul de Toulouse (CICT). 


\begin{tabular}{|c|c|c|c|c|}
\hline Téléphone & $11 \%$ & $32 \%$ & $13 \%$ & $18 \%$ \\
\hline Télécopie & $0 \%$ & $2 \%$ & $9 \%$ & $4 \%$ \\
\hline Courrier postal & $2 \%$ & $10 \%$ & $17 \%$ & $10 \%$ \\
\hline & $100 \%$ & $100 \%$ & $100 \%$ & $100 \%$ \\
\hline
\end{tabular}

Le fait que le face à face soit déclaré comme le type d'échange "le plus fréquemment le plus fréquent" avec des correspondants éloignés indique que les autres modes de communication à distance sont nettement délaissés. De fait si le téléphone est utilisé dans $53 \%$ des relations (surtout à l'intérieur du pays), le fax ne l'est que pour $22 \%$ et le courrier postal pour $37 \%$. Bien qu'on ne puisse pas effectuer de comparaison terme à terme à cause d'un mode différent de collecte et d'analyse des données, signalons quand même que l'enquête de Wellman indique un usage important du téléphone.

\section{Les caractéristiques des relations entretenues par courrier électronique}

Les correspondants se partagent entre ceux qui appartiennent au monde professionnel de l'enquêté $(72 \%)$ et ceux qui n'en font pas partie, famille ou amis d'enfance (16\%), anciens camarades d'études $(9 \%)$ ou connaissances d'un autre type $(3 \%)^{17}$.

Les relations du même monde professionnel se partagent à peu près en trois parts égales entre des relations strictement professionnelles (échanges limités à des contenus liés à l'exercice professionnel), "amicalement professionnelles" (échanges portant à la fois sur des aspects professionnels et non professionnels - le fait de rajouter dans un courrier professionnel des références à la vie de famille, la politique ou d'autres thèmes "privés" - mais sans présence d'échanges totalement non professionnels) et "amicales et professionnelles" (échanges professionnels et présence d'échanges d'autres natures sans contenu professionnel).

Les enquêteurs devaient évaluer la force des liens en fonction des réponses de l'enquêté. Cette mesure est évidemment grossière (nous avons utilisé une échelle à cinq niveaux), mais il est intéressant de constater que les liens se répartissent à peu près équitablement entre liens forts et liens faibles. On trouve plus de liens forts en dehors de l'univers professionnel (50\% pour les anciens camarades d'études, $70 \%$ pour la famille, amis d'enfance ou autres), mais un tiers des liens créés dans cet univers sont des liens plutôt forts.

\footnotetext{
17 Pour mémoire, dans les enquêtes de Wellman et Fisher la famille représente entre $40 \%$ et $50 \%$ des liens cités et les collègues de travail entre 6 et 10\%, la différence étant représentée par les voisins, membres d'une même organisation ou "juste amis". Là encore, la compraison ne peut aller très loin faute d'une référence portant sur le monde scientifique et d'informations sur les autres liens des personnes interrogées.
} 
Lors de la précédente étude nous avions demandé aux enquêtés d'évaluer la proportion de leurs correspondants rencontrés sur le réseau. Cette proportion s'établissait en moyenne à un quart. Dans notre échantillon de correspondants, il ne sont que 18 (7\%), ce qui peut s'expliquer par une surévaluation des enquêtés lors de notre précédente enquête ou par l'effet de sélection qui a conduit à prendre des correspondants relativement réguliers dans notre échantillon actuel. 10 de ces correspondants rencontrés sur le réseau résident à l'étranger et 5 en France ailleurs qu'à Toulouse. 10 d'entre eux ont été rencontrés dans un contexte professionnel. Un tiers représentent des liens plutôt forts, 7 avec des échanges au moins hebdomadaires. Dans 9 cas, il n'y a pas eu d'échange en face à face depuis le début de la relation. Il s'agit dans ce cas plutôt de liens non professionnels (la moitié) entre étudiants, soit par l'intermédiaire de jeux, soit par la participation à des groupes de discussion. Cela confirme qu'il est possible de construire un lien fort à travers le réseau ${ }^{18}$. D'un point de vue statistique toutefois, les échanges qui ne passent que par le courrier électronique à l'exclusion des autres modes de communication (que le correspondant ait été rencontré ou non sur le réseau) tendent à concerner des liens plus faibles que les autres échanges.

Il est intéressant de noter que 15 sur 18 de ces correspondants rencontrés sur le réseau sont en relation avec ceux de nos enquêtés que nous avons qualifié de "professionnels" ou "communautaires", soit les catégories rassemblant les utilisateurs les plus intensifs d'Internet. Ils sont les plus nombreux à utiliser Internet comme mode principal d'échange avec leurs correspondants (66\% des cas contre $50 \%$ pour les autres catégories) et ont d'ailleurs plus de correspondants. En regroupant ainsi nos catégories, nous retrouvons en somme l'opposition entre les "Internautes militants" et les "utilitaristes" décrits par F. Carmagnat (1996).

\section{Structure des réseaux}

L'enquête de Fisher (1982) évaluait la densité des réseaux sociaux à 0.5 environ en milieu rural à 0.4 en milieu urbain. Wellman aboutissait de son côté à une densité moyenne de 0.33 .

La mesure de densité dont nous disposons ne concerne que les personnes avec qui nos enquêtés sont en relation par courrier électronique, ce qui exclut leurs autres liens. Pour nos quinze enquêtés, la densité moyenne s'établit autour de 0.25 , et apparaît donc inférieure à la densité "habituelle" des réseaux sociaux ${ }^{19}$. Toutefois cette moyenne recouvre en fait deux groupes bien distincts. D'un côté les utilisateurs les moins intensifs (nos groupes "minimalistes", "utilitaristes" ou "contraints"), soit 7 de nos enquêtés, pour lesquels la densité moyenne s'établit autour de 0.4 avec peu de variations. De l'autre, nos 8 utilisateurs les plus intensifs avec une densité moyenne située autour de 0.15 . Tout se passe donc comme si l'usage instensif de la communication électronique entraînait l'établissement de réseaux plus faiblement denses.

Pour mieux comprendre ce point, nous pouvons donner deux exemples de réseaux typiques des deux catégories d'utilisateurs.

\footnotetext{
18 Dans les cinq cas ou aucun autre mode de communication n'a été utilisé, on trouve encore 2 liens forts. 19 Nous évaluons la densité en divisant le nombre de liens existant entre les correspondants de l'enquêté par le nombre de liens possibles entre ces correspondants.
} 
Le graphique qui suit représente les liens entre les correpondants de l'un de nos enquêtés caractérisé comme "minimaliste" (c'est-à-dire n'utilisant que le courrier électronique et non les autres aspects d'Internet). Pour chaque correspondant, nous donnons le lieu de travail ou de résidence, le statut, l'ancienneté de la relation et sa tonalité ( $\mathrm{a}=$ amical, ap $=$ amicalement professionnel, $\mathrm{p}=$ professionnel).

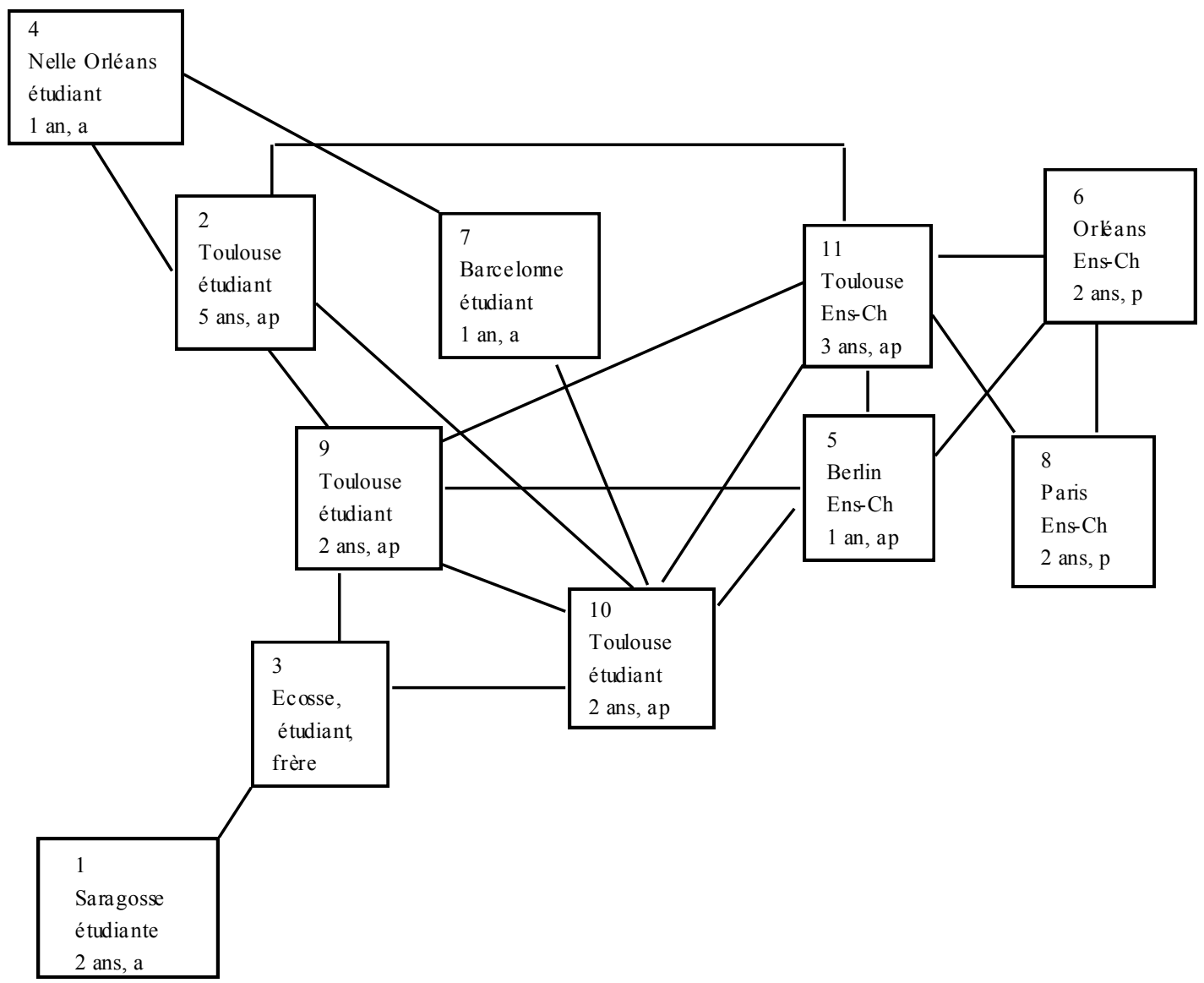

$$
\begin{aligned}
& \text { Liens entre les correspondants d'un "minimaliste" } \\
& \text { (étudiant, moins de } 30 \text { ans, densité }=0.34 \text { ) }
\end{aligned}
$$

Dans ce premier exemple, on distingue l'existence de sous-réseaux (celui des étudiants et celui des enseignants) mais ils sont reliés par de nombreux autres liens ( 5 en tout) que ceux qui passent par l'enquêté, ce qui contribue à densifier le réseau d'ensemble. À l'exception du frère de l'enquêté, lui-même étudiant, tous les correspondants sont dans l'univers "professionnel" de l'enquêté, c'est-à-dire étudiants ou enseignants-chercheurs.

Le cas suivant, fragment du réseau d'un "communautaire", enseignant-chercheur entre 40 et 50 ans, présente une structure très différente. 

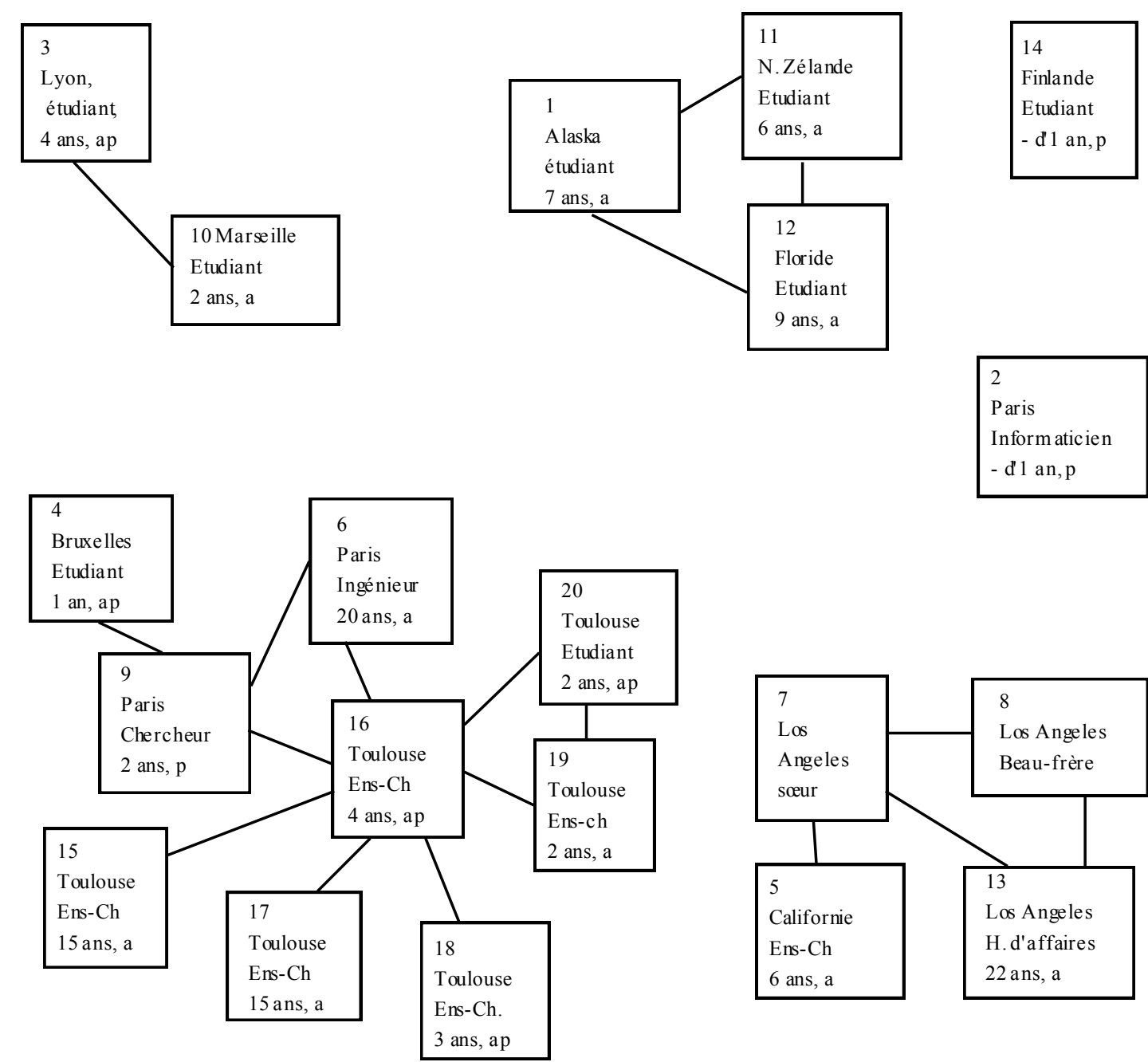

Liens entre les correspondants d'un "communautai re" (chercheur, entre 30 et 40 ans, densité $=0.12$ )

Dans ce cas apparaissent nettement plusieurs sous-réseaux totalement disjoints : le réseau professionnel, le plus important, un réseau familial et amical (la sœur, le beaufrère et deux amis habitant Los Angeles), deux petits réseaux composés d'étudiants (l'un en France, l'autre dans des pays anglophones) et enfin deux personnes isolées. Ce réseau très segmenté présente évidemment une densité très faible.

On retrouve donc l'un des résultats de Fisher et de Wellman, qui était que les réseaux à densité plus faible ne manifestaient pas une atomisation complète des relations mais un éclatement en groupes ou cercles disjoints.

Ces différents résultats nous permettent à présent de formuler une hypothèse générale sur les réseaux sociaux intégrant la communication électronique comme mode d'échange. Il ne s'agit évidemment que d'une hypothèse étant donné l'étroitesse de notre échantillon et le caractère fragmentaire et incomplet des réseaux que nous avons 
reconstitués. Néanmoins, tous nos résultats convergent vers cette hypothèse, qui restera à tester de manière plus approfondie.

\section{Conclusion : l'hypothèse de l'hyper-ville}

Il est intéressant de remarquer que de nombreux discours sur les effets d'Internet retrouvent les logiques et les arguments qui avaient cours dans les années soixante sur l'urbanisation. Certains présentent la croissance du réseau comme un accroissement de la liberté et y voient la solution de nombreux problèmes sociaux, d'autres dénoncent la régression des échanges interpersonnels, le caractère froid du médium, les possibilités de contrôle accru qu'il offre aux gouvernants de tous ordres.

De la même façon, dans les années soixante l'urbanisation et la constitution de la société de masse a suscité une abondante littérature sur la perte des liens sociaux et l'atomisation de la société qu'impliquait la concentration urbaine (Wellman, 1979, Fisher, 1982). Ce que Wellman a appelé la "question de la communauté" est restée longtemps une source de controverses au sein de la sociologie américaine, opposant les thèses de la communauté perdue, conservée, ou "libérée"20.

Les enquêtes de Fisher et Wellman visaient précisément à tester cette hypothèse sur le plan des réseaux sociaux. En comparant les réseaux en milieu rural et urbain, Fisher et son équipe ont démontré que la vie en ville ne se traduit pas par un retrécissement des relations (le volume des liens forts reste stable) mais par un déploiement différent. Les relations en ville apparaissaient comme plus ségrégatives (les jeunes fréquentant moins les vieux, les diplômés moins ceux qui ont fait peu d'études), faisant moins de part à la famille. En résumé elles apparaissaient comme plus choisies et moins subies. Sur le plan de la structure des réseaux, le paramètre marquant cette différence était la densité, plus faible en ville parce que correspondant à l'insertion dans de multiples cercles distincts. De son côté, Wellman démontrait l'existence en milieu urbain de liens intimes permettant aux acteurs de trouver du soutien, ces liens se déployant pour l'essentiel sur un espace métropolitain et non sur celui du quartier, ainsi que la persistence de liens forts entre parents et enfants. Ses conclusions diffèrent toutefois de celles de Fisher sur un point : selon lui, les réseaux en milieu urbain ne diffèrent pas seulement des anciennes "communautés" par l'existence de multiples cercles disjoints dans lesquels sont insérés les acteurs, mais aussi par leur nature, qui implique non plus une appartenance passive mais une gestion active des ressources de réseau, tout en posant des problèmes d'identité, les réseaux étant moins identifiables et identifiants que les comunautés traditionnelles.

Ce que suggèrent nos résultats, c'est que l'accès au courrier électronique et plus généralement aux services d'Internet favorise la formation et le maintien de relations qui se distinguent des relations ordinaires comme les relations en milieu urbain se différencient des relations en milieu rural (ou au sein des "communautés" au sens américain du terme) : des liens plus faibles, plus éphémères, plus disjoints, produisant des réseaux plus segmentés, à la densité plus faible. Par ailleurs, le nombre des liens, donc la taille des réseaux, semble plus élevé, puisque les usagers les plus intensifs ont plus de correspondants que les autres, bien qu'il soit difficile sur ce point d'établir une

\footnotetext{
20 Voir l'article de Wellman (1979) pour une synthèse de ces controverses.
} 
comparaison avec les données dont nous disposons, qui ne comprennent pas les relations hors réseau (rien n'exclut que les utilisateurs intensifs aient moins de relations que les autres en dehors du réseau).

En somme, l'accès généralisé à la communication électronique pourrait avoir sur les réseaux sociaux des effets comparables à ceux qu'a pu avoir l'urbanisation en renforçant la possibilité d'échanger régulièrement et facilement avec un grand nombre de personnes : ni paradis, ni enfer, mais une évolution de nos façon de vivre ensemble...

\section{Références du texte}

Bickson T.K. et Eveland J.D., 1990, "The interplay of workgroup structures ad computer support" in Galegher J., Kraut R.E. et Egido C. (eds.), Interpersonnal behaviour in small groups, Englewoods Cliffs, NJ, Prentice Hall

Bougnoux B. (dir.), 1996, Sciences de l'information et de la communication, Larousse.

Carmagnat F., "Une société électronique technicienne face à l'élargissement du réseau", Réseaux, n77, CNET, 1996Debray R., 1991, Cours de médiologie générale, Gallimard Degenne A. and Forsé M., 1994, Les Réseaux Sociaux, Paris, Armand Colin

Finholt T. , Sproull L. et Kiesler S., 1990, "Communication and performance in had hoc task groups, in J. Galegher, R.E. Kraut et C. Egido (eds.), Interpersonnal behaviour in small groups, Englewoods Cliffs, NJ, Prentice Hall.

Fisher Claude S., To dwell among friends, University of Chicago Press, 1982

Garton L. et Wellman B., 1995, "Social impacts of electronic mail in organizations : a review of the research literature", in Brant R. Burleson (ed.) Communication yearbook, $\mathrm{n}^{\circ} 18$, Thousand Oaks, CA : Sage ;

Granovetter M., 1973. "The strength of weak ties." American Journal Of Sociology, Vol. 78

Grossetti M., Sauvageot A., Branquart S., Marinesque D., Compère C., Paganelle L., Estebenet J.-P., Saint-Pierre J., 1996, "L'usage d'Internet par les chercheurs toulousains", Flux, n²4, Avril-Juin

Haythornwaite C. et Wellman B., 1996, "Which kinds of network members communicate by Email or face-to-face for what kind of work ?", University of Toronto, Centre for urban and community studies, 10p.

Hennion A., 1995, La passion musicale. Sociologie de la médiation, Metaillé

Hert P., "Les arts de lire le réseau. Un cas d'innovation technologique et ses usages au quotidien dans les sciences", Réseaux, n77, CNET, 1996

Jouët J. et Toussaint Y., 1991, "La télématique interpersonnelle ; étude du courrier électronique privé", rapport CNET/IRIS, Juin

Jouët J., 1993, "Pratiques de communication et figures de la médiation", Réseauix, n60, CNET

Latour B., "Une sociologie sans objet ? Remarques sur l'interobjectivité", Sociologie du travail, $\mathrm{n}^{\circ} 4,1994$.

Milgram S., 1967, "The small-world Problem", Psychology today, n¹

Scott J., 1992, Social Network Analysis, Newbury Park CA, Sage

Wasserman S. and Faust K., 1994, Social Network Analysis, Cambridge, Cambridge University Press ; Wellman B. and Berkowitz S.D., 1997, Social Structures : A Network Approach. Greenwich, CT JAI Press. 
Wellman B. et Gulia M., 1996 "Net surfers don't ride alone : virtual communities as communities", in P. Kollock and M. Smith (eds.), Communities in cyberspace, Berkeley, University of California Press.

Wellman B., 1992, "Men in networks : private communities, domestic friendships", in P. Nardi (ed.), Men's friendships, Newbury Park, CA : Sage

Wellman B., 1996, "An electronic group is virtually a social network" in sara Kiesler (ed.), The culture of the Internet, Hillsdale, NJ : Lawrence Erlbaum.

Wellman B., Salaff J., Dimitrova D., Garton L., Gulia M., Haythornwaite C., 1996, "Computer networks as social networks : collaborative work, telework, and virtual community", Annual review of Sociology, $\mathrm{n}^{\circ} 22$

Wellman B., 1979, "The Community Question : The Intimate Networks of East Yorkers", American Journal of Sociology, vol. 4, n5 